\title{
Desenvolvimento de aplicativo móvel para monitorização dos níveis glicêmicos obtidos por medições capilar e sensor subcutâneo
}

\author{
Development of a mobile application for monitoring glycemic levels obtained by capillary \\ measurements and subcutaneous sensor \\ Desarrollo de una aplicación móvil para el seguimiento de los niveles glucémicos obtenidos
} mediante medidas capilares y sensor subcutâneo

Recebido: 29/03/2021 | Revisado: 05/04/2021 | Aceito: 13/04/2021 | Publicado: 24/04/2021

Rosângela Simões Gonçalves
ORCID: https://orcid.org/0000-0001-6605-6574
Instituto Nacional de Saúde da Mulher, da Criança e do Adolescente Fernandes Figueira, Brasil
Centro Universitário Cesmac, Brasil
E-mail: rosangelasimoes.hu @ hotmail.com
Guilherme Benjamin Brandão Pitta
ORCID: https://orcid.org/0000-0002-8917-5442
E-mail: guilhermebbpitta@gmail.com
Roseli de Souza Santos da Costa
Universidade Estadual de Cências da Saude de Alagoas, Brasil
ORCID: https://orcid.org/0000-0001-5671-828X
E-mail: roseli.costa@iff.fiocruzibr
Felipe Roberto Eloi Moura
Instituto Nacional de Saúde da Mulher, da Criança e do Adolescente Fenandes Figuira, Brasi
ORCID: https://orcid.org/0000-0002-7509-1942
Universidade Federal de Alagoas, Brasil
E-mail: felipemoura.al@gmail.com
Simone Regina Alves de Freitas Barros
ORCID: https://orcid.org/0000-0003-0353-8546
Centro Universitário Tiradentes de Maceió, Brasil
E-mail: simoninhabarros2010@ @hotmail.com
Caroline Simões Gonçalves Castilho
ORCID: https://orcid.org/0000-0001-7842-4813
Centro Universitário Cesmac, Brasil
E-mail: caroline.csgc@ @otmail.com
Daniel Simões Gonçalves Castilho
ARCID: https://orcid.org/0000-0003-0450-8701
Centro Universitário Cesmac, Brasil
E-mail: daniels1312@outlook.com
ORCID: https://orcid.org/0000-0002-9268-6921
Universidade Federal de Alagoas, Brasil
E-mail: ailton_fisioterapeuta@ hotmail.com

\section{Resumo}

Um dos desafios para os pacientes com diabetes mellitus (DM) é controlar as concentrações glicêmicas e, observa-se melhora nos resultados clínicos quando utilizam aplicativos móveis para realizar o seu autocuidado. A monitorização da glicemia por técnica capilar (GC) fornece apenas dados intermitentes do controle metabólico, impedindo uma visão completa do perfil glicêmico do paciente. Com a Monitorização Contínua de Glicose (CGM), é possível avaliar com exatidão as variações glicêmicas ao longo do dia, com intervenção terapêutica mais eficaz. Diante destas premissas a presente pesquisa teve como objetivo promover o desenvolvimento de um aplicativo móvel, denominado UP GLICEMIA, para monitorização da glicose por CGM por meio do sensor subcutâneo e GC, que geram relatórios gráficos simultâneos. Os valores glicêmicos, quando medidos e registrados no aplicativo, são informados ao usuário em forma de padrões de alertas para normoglicemia, tolerável, hiperglicemia e hipoglicemia, apresentados em cores verde, amarelo, vermelho e roxo, respectivamente. O referido aplicativo, também gera relatórios gráficos diários, semanais e mensais para as duas técnicas de medidas glicêmicas por meio de gráficos individuais e também 
associados, que poderão auxiliar na conduta terapêutica. Tratou-se de uma pesquisa aplicada, exploratória para desenvolvimento de aplicativo, com uma nova perspectiva para o controle glicêmico. Espera-se que a utilização do UP GLICEMIA para o paciente com DM tipo 1 e tipo 2 e pelo profissional de saúde possa auxiliar no melhor entendimento do monitoramento da glicose do usuário, demonstrando o perfil glicêmico por completo e possibilite o controle da glicemia.

Palavras-chave: Glicemia; Diabetes mellitus; Aplicativo móvel.

\begin{abstract}
One of the challenges for patients with diabetes mellitus (DM) is to control glycemic concentrations and, there is an improvement in clinical results when using mobile applications to perform their self-care. Blood glucose monitoring by capillary technique provides only intermittent data on metabolic control, preventing a complete view of the patient's glycemic profile. With Continuous Glucose Monitoring (MCG), it is possible to accurately assess glycemic variations throughout the day, with more effective therapeutic intervention. Given these premises, this research aimed to promote the development of a mobile application, called UP GLICEMIA, for glucose monitoring by CGM through the subcutaneous sensor and GC, which generate simultaneous graphical reports. The glycemic values when measured and registered in the application, are informed to the user in the form of alert patterns for normoglycemia, tolerable, hyperglycemia and hypoglycemia, presented in green, yellow, red and purple, respectively. This application also generates daily, weekly and monthly graphical reports for the two techniques of glycemic measurements by means of individual and associated graphs, which may assist in the therapeutic conduct. It was an applied, exploratory research for application development, with a new perspective for glycemic control. It is expected that the use of UP GLICEMIA for patients with type 1 and type $2 \mathrm{DM}$ and by the health professional can help in a better understanding of the glucose monitoring of the user, demonstrating the glycemic profile completely and enabling glycemic control.

Keywords: Glucose; Diabetes mellitus; Mobile application.
\end{abstract}

\begin{abstract}
Resumen
Uno de los retos para los pacientes con diabetes mellitus (DM) es controlar las concentraciones glucémicas y, hay una mejora en los resultados clínicos al utilizar aplicaciones móviles para realizar su autocuidado. La monitorización de la glucosa en sangre mediante la técnica capilar proporciona solo datos intermitentes sobre el control metabólico, lo que evita una visión completa del perfil glucémico del paciente. Con la monitorización continua de glucosa (MCG), es posible evaluar con precisión las variaciones glucémicas a lo largo del día, con una intervención terapéutica más eficaz. Dadas estas premisas, esta investigación tuvo como objetivo promover el desarrollo de una aplicación móvil, denominada UP GLICEMIA, para la monitorización de glucosa por CGM a través del sensor subcutáneo y CG, que generen informes gráficos simultáneos. Los valores glucémicos medidos y registrados en la aplicación, son informados al usuario en forma de patrones de alerta por normoglucemia, tolerable, hiperglucemia e hipoglucemia, presentados en verde, amarillo, rojo y morado, respectivamente. Esta aplicación también genera informes gráficos diarios, semanales y mensuales de las dos técnicas de medición glucémica mediante gráficos individuales y también asociados, que pueden ayudar en la conducta terapéutica. Fue una investigación exploratoria aplicada para el desarrollo de aplicaciones, con una nueva perspectiva para el control glucémico. Se espera que el uso de UP GLICEMIA para pacientes con DM tipo 1 y tipo 2 y por parte del profesional de la salud pueda ayudar a una mejor comprensión del control de glucosa del usuario, demostrando el perfil glucémico por completo y permitiendo el control glucémico.
\end{abstract}

Palabras clave: Glucosa; Diabetes mellitus; Aplicación movil.

\title{
1. Introdução
}

Um dos desafios para os pacientes com diabetes é ter o controle das suas concentrações glicêmicas. Tem-se observado melhora nos resultados clínicos de portadores de diabetes que utilizam aplicativos móveis para melhorar o autocuidado do Diabetes Mellitus (DM) (Nundy et al., 2013). Nesse contexto, o uso de aplicativos móveis que mantenham registros sobre a alimentação, histórico dos níveis de glicose no sangue, interação medicamentosa, atividade física bem como o modo como o paciente está se sentindo pode ser um forte aliado para o gerenciamento do DM (Sociedade Brasileira de Diabetes [SBD], 2018).

O desenvolvimento tecnológico vem acontecendo rapidamente e se estende a dispositivos móveis como celulares, smartphones e tablets. Nesse sentido, diversos softwares para todas as áreas da saúde estão sendo criados e/ou aperfeiçoados. A utilização de tecnologia digital para cuidados em saúde constitui um importante recurso para auxiliar profissionais e indivíduos, sendo uma potencial ferramenta nas práticas médicas e de saúde pública, na medida em que colabora para a 
melhoria da gestão da informação, o acesso aos serviços, a qualidade do cuidado prestado e a contenção de custos (Curioni et al., 2013),além de possibilitar uma oportunidade singular para a promoção de estilo de vida saudável, o tratamento e a prevenção de doenças crônicas (Guillén et al., 2009).

O uso de tecnologias informatizadas tem sua incorporação emergente e já são reconhecidas na literatura como uma ferramenta que otimiza o processo de gerenciamento, cuidado, ensino e pesquisa, estando diretamente relacionada com a melhora da qualidade dos serviços de saúde prestados (Goyatá et al., 2012; Queiroz et al., 2012).

Duas técnicas primárias estão disponíveis para profissionais de saúde e pacientes realizarem excursões glicêmicas e seu controle: automonitorização da glicose no sangue, obtida pela Glicemia Capilar (GC) e a mensuração da glicose intersticial pelo sistema de Monitorização Contínua de Glicose (Continuos Glucose Monitoring - CGM) sendo ambas realizadas no domicílio do paciente (Alencar, Muniz \& Medeiros, 2018).

A monitorização do DM por técnica capilar fornece apenas dados intermitentes do controle metabólico, impedindo uma visão completa do perfil glicêmico do paciente (Ferraz, Maia \& Araújo, 2004). Com a CGM, tem sido possível avaliar com exatidão as variações glicêmicas ao longo do dia, com intervenção terapêutica mais eficaz e redução da hemoglobina glicada (A1c) (Kaufman, 2000; Boland \& Tamborlane, 2000).

Observa-se na literatura que vários tipos de aplicativos vêm sendo desenvolvidos para auxiliar os pacientes no tratamento do DM Tipo 1 e Tipo 2 (Bellei et al.,2018). Nesse sentido, o UP GLICEMIA, é um aplicativo para Smartphone que fornece dados relacionados ao autocuidado com DM.

Diante da importância da utilização de aplicativos móveis para melhorar a monitorização da glicemia em indivíduos portadores de DM, surgiu o interesse de criação de uma ferramenta informatizada, que possibilitasse ao usuário fazer a monitorização dos níveis glicêmicos por medições realizadas por GC e por CGM (não intermitente), sendo esses verificados, respectivamente, pelo glicosímetro e o sensor subcutâneo.

O interesse em desenvolver o aplicativo UP GLICEMIA, para a monitorização das duas técnicas deveu-se ao fato da inovação tecnológica do sensor subcutâneo poder identificar picos glicêmicos nas variações intermediárias da glicose em relação às medidas de GC, que não capturam os picos glicêmicos nas flutuações e na mesma frequência favorecendo, dessa forma, o controle glicêmico dos portadores de DM tipo 1 e tipo 2 que realizam o automonitoramento.

Assim, o presente estudo teve como foco o desenvolvimento de um aplicativo móvel, denominado UP GLICEMIA, para monitorização da glicose por CGM por meio do sensor subcutâneo e GC, gerando relatórios gráficos simultâneos.

\section{Fundamentação Teórica}

\subsection{Diabetes Mellitus}

O termo Diabetes Mellitus (DM) é definido como uma síndrome de etiologia múltipla e pode ser ocasionado pela falta de insulina e/ou incapacidade da insulina exercer adequadamente suas funções (S, 2016). A hiperglicemia ou o aumento do nível de açúcar no sangue é um efeito comum da DM não controlada e, ao longo do tempo, causa sérios danos a muitos dos sistemas do corpo, especialmente os nervos e os vasos sanguíneos (Organização Mundial da Saúde [OMS], 2017).

O diabetes compreende diversos distúrbios caracterizados por hiperglicemia. De acordo com a atual classificação, existem dois tipos principais: Diabetes tipo 1 (DM1) e Diabetes tipo 2 (DM2). Historicamente, a diferenciação entre os dois tipos tem sido baseada na idade de início, no grau de perda da função de células $\beta$, grau de resistência à insulina, presença de autoanticorpos associados ao diabetes e necessidade de tratamento com insulina para sobrevivência (Leslie et al., 2016).

O DM1 é uma doença autoimune, poligênica, decorrente de destruição das células $\beta$ pancreáticas, ocasionando deficiência completa na produção de insulina, com a prevalência de 10\% dos casos (Insel et al., 2015; Chiang et al., 2014). O 
DM2 é uma doença crônica, apresentando como principal fenômeno fisiopatológico a resistência à ação da insulina, diminuindo a captação de glicose em tecidos insulino dependentes (Ferreira et al., 2011; Geloneze, Lamounier \& Coelho, 2006).

O DM em estágios iniciais pode ser oligossintomático ou até assintomático, o que retarda seu diagnóstico, aumentando o risco para complicações agudas, entre elas a hipoglicemia, a cetoacidose diabética e o coma hiperosmolar; e crônicas, como as alterações micro e macrovasculares (Queiroz et al., 2011). O DM pode apresentar sintomas característicos como perda de peso, visão embaçada, sede e poliúria. Manifestações clínicas mais graves como cetoacidose ou um estado hiperosmolar não cetótico que podem levar à desidratação, coma e, na ausência de tratamento efetivo, morte. Além disso, infecções genitais por leveduras 25 ocorrem com frequência. Estima-se que uma porcentagem significativa de casos de diabetes (30-80\%, dependendo do país) não são diagnosticados (Zimmet, Alberti \& Shaw, 2001).

O aumento da prevalência do diabetes está associado a diversos fatores, como: maior frequência de estilo de vida sedentário, transição nutricional, rápida urbanização, transição epidemiológica, maior frequência de excesso de peso, crescimento e envelhecimento populacional e, também, à maior sobrevida dos indivíduos com diabetes (S, 2017). A IDF, em 2017, estimou que 8,8\% a população mundial entre 20 e 79 anos de idade (426 milhões de pessoas) vivia com diabetes, ressaltando que se as tendências atuais persistirem, o número de pessoas com diabetes foi projetado para ser superior a 629 milhões em 2045 (SBD, 2020).

\subsection{Tipos de aplicativos que relacionam o Diabetes Mellitus e a saúde}

Existem evidências, ainda que preliminares, que há melhor controle glicêmico associado ao uso de aplicativos móveis em saúde. De acordo com Arrais e Crotti (2015) as perspectivas são claramente animadoras, pela convergência de fatores atualmente observada, ou seja, aumento da demanda pelos serviços sendo acompanhada pela oferta de soluções cada vez mais integradas e com interface homem-máquina progressivamente mais intuitivas e dinâmicas e progressivamente mais acessíveis, mesmo em populações com menor renda.

Até 2013 já havia mais de 31.000 apps destinados a cuidados com a saúde, apenas considerando as plataformas Android (sistema operado pela empresa Google Inc.) e iOS (sistema operado pela Apple Inc.) (Arnhold, Quade \& Kirch, 2014). Destes aplicativos, uma parcela considerável e crescente (Figura 1) tem sido desenvolvida para ajudar pacientes no tratamento do diabetes mellitus tipo 1 ou 2 (Martinez-Pérez, De La Torre-Diéz \& López-Coronado, 2013; Chomutare et al., 2011).

Figura 1 - Evolução de aplicativos para diabetes (Android/iOS).

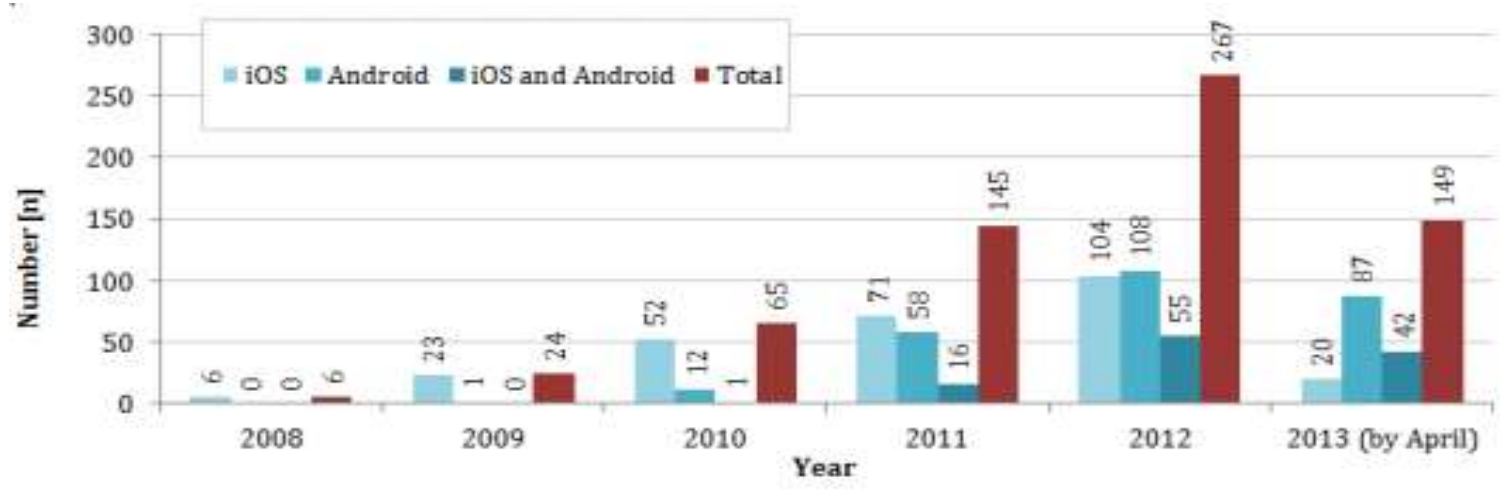

Fonte: Madlen Arnhold et al. (2014). 
Entre os aplicativos que relacionam o DM e a saúde pode-se citar: o OnTrack Diabetes, desenvolvido pela Medivo (2015); o Diabetes M de Rossen e Varbanov (2015) e o Diabetes Plus oferecido pela SquareMed Software GmbH (2015), o Gliconline desenvolvido pela Quasar Telemedicina (2014) bem como o My Sugar e o FreeStyler Libre Link, desenvolvido pela Abbott. A seguir são descritos as principais características desses aplicativos.

- OnTrack Diabetes: se propõe a auxiliar profissionais e pacientes no gerenciamento e controle do diabetes por meio do acompanhamento de estatísticas como glicemia, hemoglobina glicada, exercícios, medicamentos e alimentos. Além disso, possibilita a geração de gráficos e relatórios detalhados com base nos dados inseridos para o compartilhamento com a equipe médica (Medivo, 2015).

- Diabetes M e o Diabetes Plus: visam a auxiliar os indivíduos no acompanhamento e na análise e manutenção do controle glicêmico (Baldo et al., 2015). Esse aplicativo permite o lançamento dos alimentos consumidos no dia, realizando posteriormente o cálculo da contagem dos carboidratos e demonstrando a quantidade exata de insulina a ser administrada pelo paciente. A ideia é manter, com isso, a glicemia dentro dos limites convenientes recomendados para diabetes tipo 1, tipo 2 ou gestacional.

- Gliconline: reconhecido internacionalmente como a melhor ferramenta para administrar o tratamento do diabetes, é um aplicativo que visa a auxiliar o controle do diabético do tipo 1 e 2 , e também ajudar médicos e nutricionistas, que podem acompanhar à distância a evolução do paciente, por meio de um prontuário virtual, ajustando a terapia quando necessário (Quasar Telemedicina, 2014). Esse aplicativo permite o cálculo e o registro das calorias e nutrientes dos alimentos informados, lembra o paciente dos medicamentos e doses a serem tomadas no horário ideal bem como calcula a dose de insulina para correção da glicemia (Quasar Telemedicina, 2014).

- MySugar: trata-se de um diário de bordo composto de um sistema de motivação, para o usuário, fazendo com que ganhe pontos para cada entrada, ajudando a atingir metas pessoais de alimentação. Dentre suas funções ele é capaz de guardar informações que são inseridas pelos usuários como os níveis de glicemia, ingestão de carboidratos, unidades de insulinas, entre outras. $\mathrm{O}$ aplicativo possui uma interface intuitiva tornando fácil o aprendizado do usuário quanto ao uso do aplicativo. Este aplicativo encontra-se disponível para iOS e Android (MySugar GMBH, 2018).

- FreeStyle Libre Link: para indivíduos portadores de Diabetes Mellitus que usam o sensor, realizar a varredura do sensor a cada 8 horas. Precisa ter acesso a um monitoramento de glicose, portanto o aplicativo não obtém tal sistema (Abbott, 2016).

\section{Metodologia}

Tratou-se de uma pesquisa aplicada, exploratória para desenvolvimento de um aplicativo, com uma nova perspectiva para o controle glicêmico, com o intuito de auxiliar os pacientes com DM Tipo 1 e Tipo 2 e o profissional de saúde em sua conduta terapêutica. A metodologia de desenvolvimento do software foi baseada no Extreme Programming. O aplicativo UP Glicemia apresenta-se em conformidade com as Diretrizes propostas pela SBD (SBD, 2017/2018) e posicionamento oficial da SBD (SBD, 01/2019).

As fases do desenvolvimento da pesquisa (Figura 5) para a criação do aplicativo foram: a pesquisa bibliográfica, levantamento de aplicações, por meio de busca manual nas lojas App Store e Play Store, requisitos, mapa conceitual do dispositivo para prototipagem e desenvolvimento de software, com intuito do aplicativo monitorizar os níveis glicêmicos por medidas das técnicas: capilar e do sensor subcutâneo. 
Figura 2 - Fases do desenvolvimento da pesquisa.

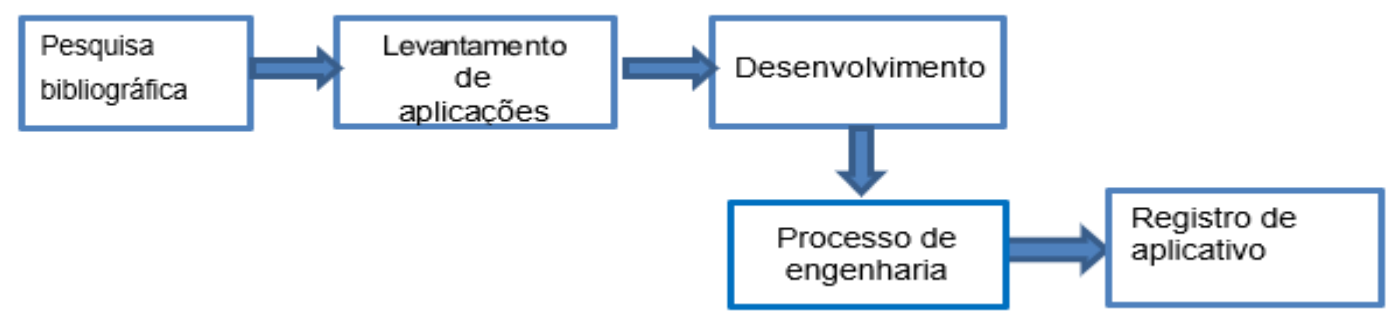

Fonte: Adaptado de Maia et al. (2018).

O levantamento teórico por indexadores Pubmed, Scientific Electronic Library Online (Scielo) e Literatura LatinoAmericana e do Caribe em Ciências da Saúde (Lilacs) foi necessário para o desenvolvimento da pesquisa, auxiliando também na formalização dos requisitos. Para o desenvolvimento do referencial teórico da pesquisa foram analisadas 34 publicações a partir de 1992 até 2020.

O levantamento de aplicativos foi realizado por busca manual nas lojas App Store e Play Store para dispositivos móveis com usabilidade no indivíduo diabético. A seleção obedeceu à lógica relacionada as palavras-chave: diabetes, glicemia, glicose, aplicativo móvel.

O dispositivo móvel desenvolvido, denominado UP GLICEMIA, aplicou uma metodologia que apresenta agilidade no desenvolvimento de software baseada em Extreme Programming, o fluxo é apresentado na Figura 4 (Pressman, 2011, p. 87). É um desenvolvimento de software que apresenta rapidez, produtividade, qualidade, atendendo à necessidade do cliente de forma simples (Bessa, 2018).

O aplicativo desenvolvido possui o sistema operacional híbrido, e pode ser publicado nas plataformas Android, iOS, Windows Mobile, sendo a plataforma Android, a que foi a utilizada para o referido aplicativo. O aplicativo irá apresentar um comportamento muito proveitoso na economia de memória para os usuários, pois, além de reduzir a quantidade de telas com a utilização de parâmetros, os dados serão salvos na nuvem.

$\mathrm{O}$ pedido de registro de programa de computador no Instituto Nacional da Propriedade Industrial (INPI) para o aplicativo UP GLICEMIA foi realizado sob o número do processo: 512020000132-7.

\section{Resultados}

Para o desenvolvimento do aplicativo foi realizada a estrutura de navegação (prototipagem), que constou com os requisitos funcionais para cadastro, login, home da área logada (medições e relatórios) e não funcionais que é a interface do aplicativo. Diante do exposto, aplicou-se a metodologia de desenvolvimento de software, o Extreme Programming, para fundamentar a construção do protótipo para oferecer melhor qualidade de usabilidade e conteúdo para o usuário.

O aplicativo UP GLICEMIA é híbrido, podendo ser desenvolvido para qualquer sistema operacional e foram utilizados em seu desenvolvimento ferramentas e linguagens como o Application Programming Interfaces (API), do Apache Cordova e as linguagens HTML, CSS e JavaScript para dispositivos portáteis da plataforma Android.

Por considerar a importância da proteção deste aplicativo já foi realizado, em 22/01/2020, o devido registro no Instituto Nacional da Propriedade Industrial (INPI) sob o número 512020000132-7.

A seguir, serão apresentadas as telas do aplicativo móvel proposto: Na tela da Figura 3 encontra-se o cadastro do aplicativo UP GLICEMIA contendo as informações que incluem: nome, sexo, tipo de diabetes, data de nascimento, e-mail, senha e confirmar senha e a política de privacidade. 
Figura 3 - Tela de Cadastro do usuário no UP GLICEMIA.

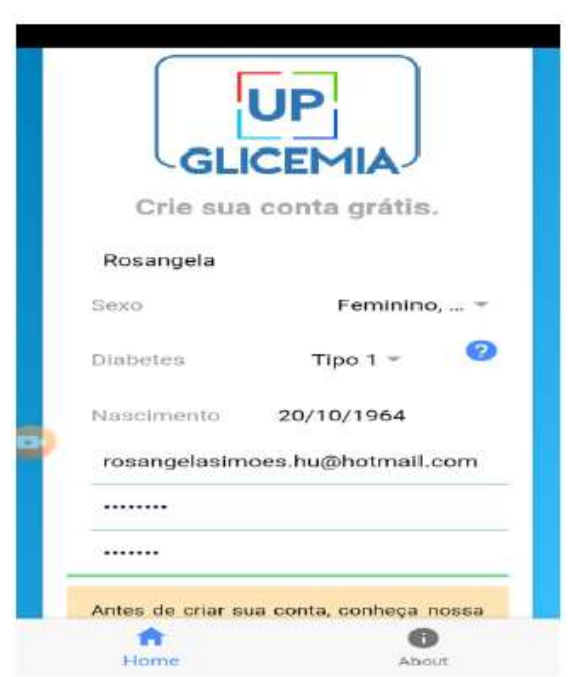

Fonte: Autores (2021).

A tela da Figura 4 representa o início da funcionalidade do aplicativo quanto aos valores glicêmicos e relatórios gráficos (diário, semanal e mensal). O botão verde localizado na parte superior da tela faz o retorno ao login e perfil do usuário.

Figura 4 - Tela de medidas glicêmicas e relatórios gráficos.

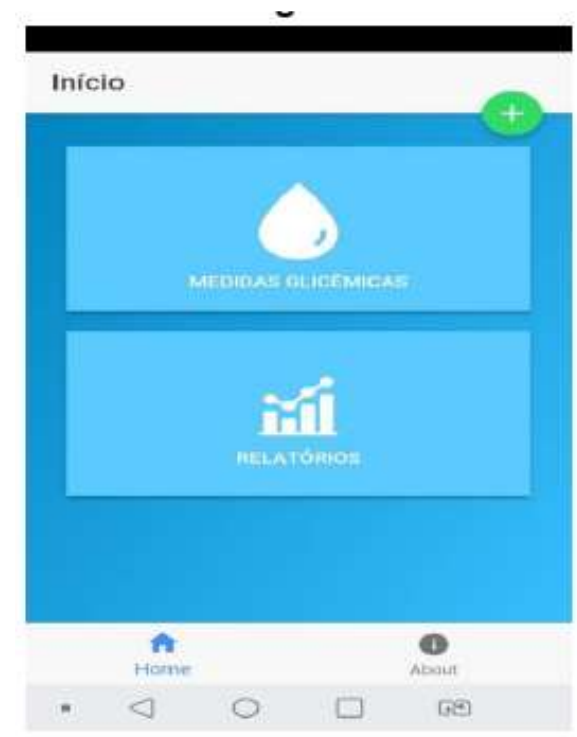

Fonte: Autores (2021).

A tela da Figura 5 contém a escolha da técnica para medições de glicose a ser utilizada pelo usuário. Na mesma tela, há o informativo quanto às metas de controle glicêmico. Os valores glicêmicos apresentados são os recomendados pela Sociedade Brasileira de Diabetes (SBD, 2019). 
Figura 5 - Tela para escolher a técnica de medidas de glicose.

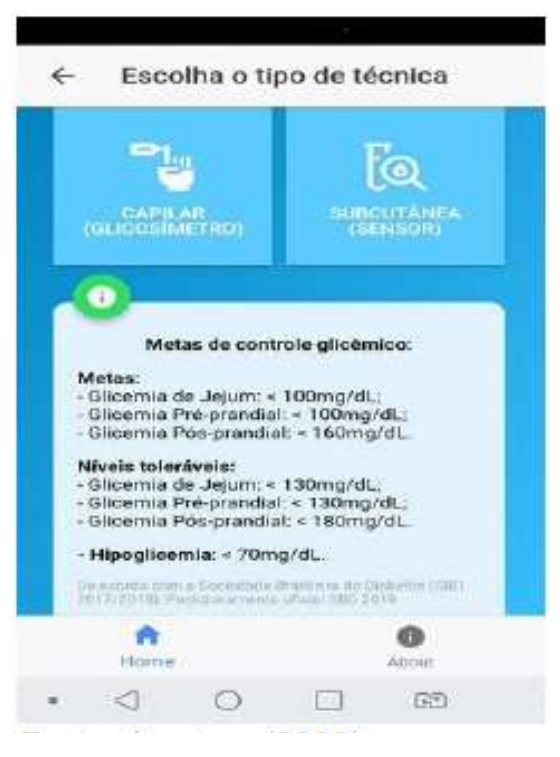

Fonte: Autores (2021).

A tela da Figura 6 representa o momento em que o usuário realizou a medição pela técnica de monitorização contínua da glicose pelo sensor subcutâneo. Após registrar o valor glicêmico medido e eventos diários, o usuário deverá teclar no botão avançar para dar continuidade ao uso do aplicativo.

Figura 6 - Tela de registro de valor glicêmico e eventos diários.

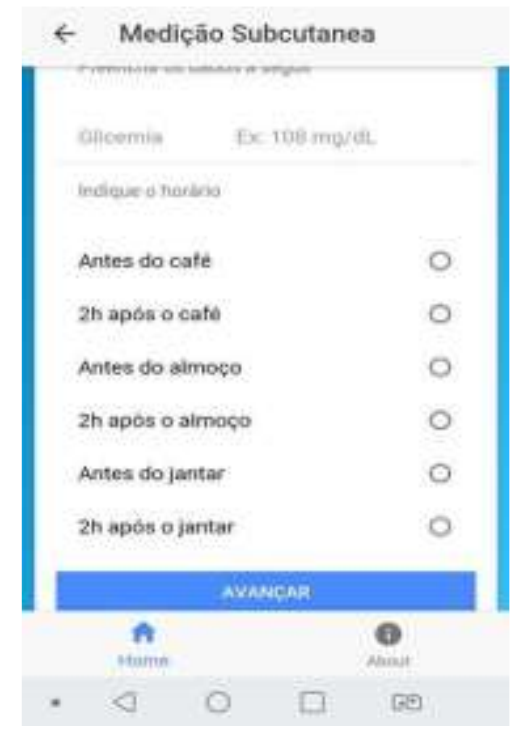

Fonte: Autores (2021).

As telas apresentadas na Figuras 7 representam a leitura para os valores glicêmicos quando medidos e registrados para as medições por duas técnicas: capilar e monitorização contínua da glicose, pelo sensor subcutâneo. As cores verde, amarelo, vermelho e roxo apresentam como sinal de alerta para demonstrar a situação da glicose no momento medido, quanto a normoglicemia, tolerável, hiperglicemia e hipoglicemia, respectivamente. 
Figura 7 - Telas de padrão de alerta para medidas capilar.

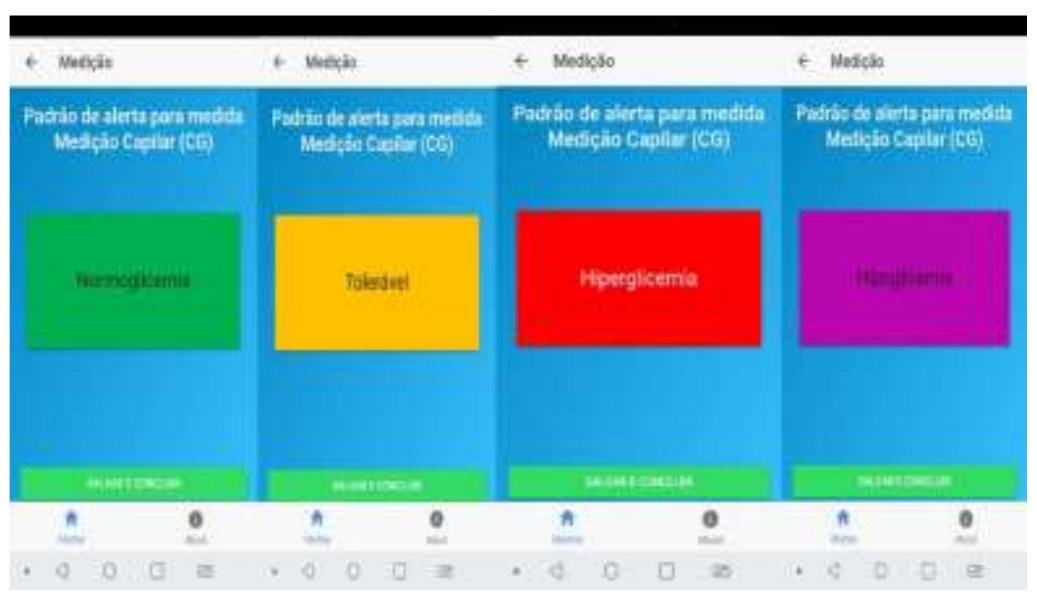

Fonte: Autores (2021).

O usuário, após verificar a informação da situação de seu valor glicêmico pelo padrão de alerta, deverá retornar para a tela de medidas glicêmicas e relatórios gráficos e optar por gerar os relatórios gráficos: diário, semanal e mensal, conforme a técnica a ser escolhida.

A tela da Figura 8 representa o relatório gráfico para os registros das duas técnicas: monitorização contínua da glicose e capilar, que são realizadas pelo sensor e o glicosímetro, respectivamente. O gráfico diário é gerado de forma simultânea para as duas técnicas, demonstrando o completo perfil glicêmico e diagnosticando pelo CGM os picos glicêmicos, em todas as variações intermediárias. Ao clicar no botão azul ou laranja, os gráficos poderão ser alternados, e apresentados individualmente.

Figura 8 - Tela do gráfico diário capilar e sensor.

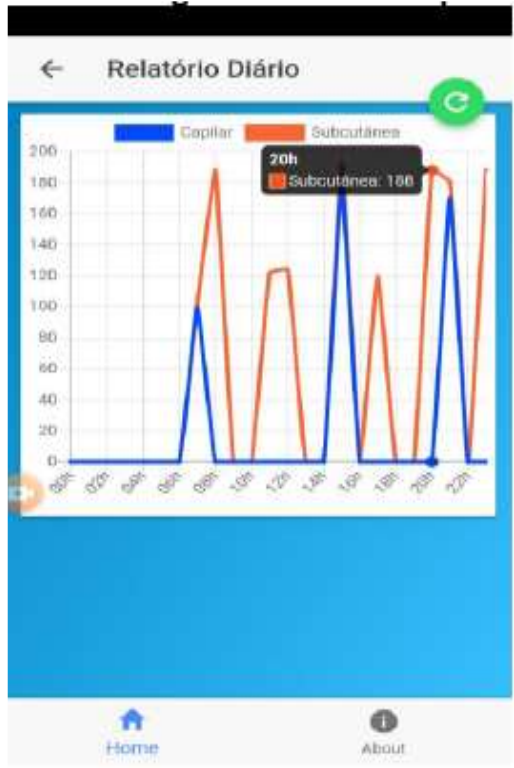

Fonte: Autores (2021).

Na tela da Figura 9 o usuário obtém o resultado do relatório gráfico mensal para as medições glicêmicas: capilar e sensor subcutâneo simultaneamente. Ao clicar o botão azul ou laranja os gráficos poderão ser apresentados individualmente. 
Figura 9 - Tela do gráfico mensal capilar e sensor.

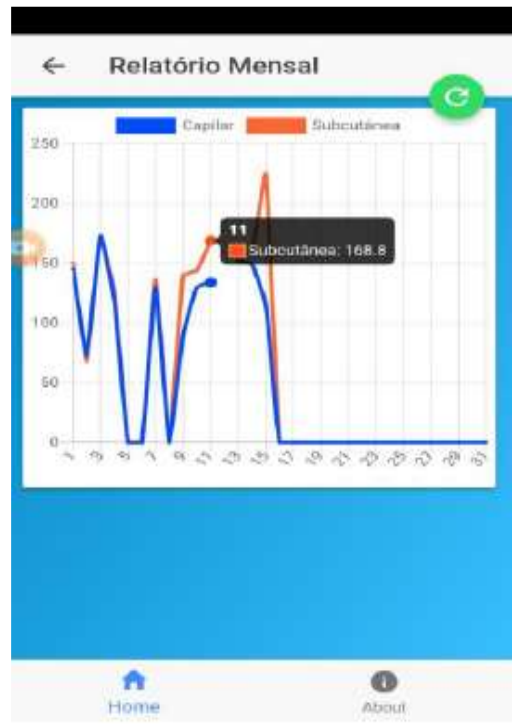

Fonte: Autores (2021).

\section{Discussão}

Os aplicativos móveis visam a atender o acesso das pessoas à informação e ao conhecimento, sem restrição de tempo e espaço (Barra et al., 2017).

O UP GLICEMIA foi desenvolvido diante do conhecimento que a GC fornece apenas dados dos níveis glicêmicos em tempo real do controle metabólico, impedindo a visão das flutuações glicêmicas que ocorrem no portador de DM (Ferraz, Maia \& Araújo, 2004) e o CGM possibilita avaliar, continuamente, as variações intermediárias glicêmicas ao longo do dia (Kaufman, 2000; Boland \& Tamborlane, 2000). O aplicativo UP GLICEMIA demonstra as variações intermediárias dos níveis glicêmicos.

A inovação tecnológica do UP GLICEMIA que o diferencia dos demais aplicativos disponibilizados no Google Play deve-se ao fato de esse ter sido desenvolvido para registrar o valor glicêmico medido para cada uma das técnicas, apresentar sinal de alerta em cores com as nomenclaturas (normoglicemia, glicemia tolerável, hiperglicemia e hipoglicemia), gerar relatório gráfico simultâneo para medida capilar e monitorização contínua da glicose, bem como, informar metas do controle glicêmico, como pode ser observado no Quadro 1. 
Quadro 1 - Comparação das funcionalidades do aplicativo móvel UP GLICEMIA com aplicativos de diabetes disponibilizados no Google Play.

\begin{tabular}{|c|c|c|c|c|c|c|c|}
\hline \multirow[b]{2}{*}{ FUNCIONALIDADE } & \multicolumn{7}{|c|}{ APLICATIVOS MOBILE } \\
\hline & $\begin{array}{l}\text { FreeStyle } \\
\text { Libre Link }\end{array}$ & Diabetes: $\mathrm{M}$ & Gliconline & OnTrack & MySugr & \begin{tabular}{|c|}
$\begin{array}{c}\text { Diabetes } \\
\text { Plus }\end{array}$ \\
\end{tabular} & $\begin{array}{c}\text { UP } \\
\text { GLICEMIA }\end{array}$ \\
\hline Cadastro de dados pessoais & $x$ & $x$ & $x$ & $\mathrm{x}$ & - & - & $x$ \\
\hline Senha para acessar o app & - & - & $\mathrm{x}$ & $\mathrm{x}$ & - & - & $\mathrm{x}$ \\
\hline $\begin{array}{l}\text { Cadastro de dados } \\
\text { glicêmicos }\end{array}$ & $\mathrm{x}$ & $\mathrm{x}$ & $\mathrm{x}$ & $\mathrm{x}$ & $\mathbf{x}$ & $\mathrm{x}$ & $\mathrm{x}$ \\
\hline $\begin{array}{l}\text { Sinal de alerta em cores, } \\
\text { com nomenclaturas: } \\
\text { normoglicêmia, tolerável, } \\
\text { hiperglicemia e } \\
\text { hipoglicemia. }\end{array}$ & - & - & - & - & - & - & $\mathrm{x}$ \\
\hline \begin{tabular}{|l|} 
Relatório gráfico para \\
medições glicêmicas
\end{tabular} & $\mathrm{x}$ & $\mathrm{x}$ & $\mathrm{x}$ & $\mathrm{x}$ & - & $x$ & $\mathrm{x}$ \\
\hline $\begin{array}{l}\text { Relatório gráfico } \\
\text { simultâneo para medida } \\
\text { capilar e monitorização } \\
\text { contínua da glicose }\end{array}$ & - & - & - & - & - & - & $\mathrm{x}$ \\
\hline $\begin{array}{l}\text { Leitura dos gráficos de } \\
\text { forma ágil e explicativa }\end{array}$ & $\mathrm{x}$ & - & - & - & - & - & $\mathrm{x}$ \\
\hline $\begin{array}{l}\text { Informativo: metas do } \\
\text { controle glicêmico }\end{array}$ & - & - & - & - & - & - & $x$ \\
\hline $\begin{array}{l}\text { App para o Diabetes tipo } 1 \\
\text { e tipo } 2\end{array}$ & $x$ & $\mathbf{x}$ & $\mathrm{x}$ & $\mathrm{x}$ & $\mathrm{x}$ & $\mathrm{x}$ & $\mathrm{x}$ \\
\hline
\end{tabular}

Fonte: Autores (2021).

Tibes, Dias, Zem-Mascarenhas (2014); Oliveira, Costa (2012) e Catalan et al. (2011) destacam que elaborar um aplicativo de maneira coerente e adequada é primordial, além de reconhecer as necessidades do usuário final, para que assim o desenvolvimento seja de acordo com as demandas específicas, testadas na pesquisa e implementadas na prática.

A utilização da tecnologia para monitorar, promover cuidados e maior adesão aos tratamentos de saúde facilita a maior integração entre equipe multiprofissional e usuário/paciente. Guillén et al. (2009) destacam que o uso dessa tecnologia possibilita uma oportunidade singular para a promoção de estilo de vida saudável, o tratamento e a prevenção de doenças crônicas e valoriza sua iniciativa que atingem simultaneamente um grande público além de aumentar a capacidade de personalização das necessidades individuais de saúde.

De acordo com Hsu et al. (2016), cuidar de doenças crônicas como o DM requer um modelo diferente, no qual os pacientes possam ser fortalecidos por meio do autocuidado e, por intermédio de uso de aplicativos móveis que mantém registros sobre a alimentação, histórico dos níveis de glicose no sangue, interação medicamentosa, atividade física e o modo como o paciente está se sentindo, isso pode ser realizado (SBD, 2018).

Diante do exposto, o UP GLICEMIA vem a ser uma ferramenta a mais para o paciente com DM tipo 1 e 2 pois é de fácil manuseio, compreensão e agilidade, além de auxiliá-lo a identificar comportamentos de riscos, permitir um autocuidado apoiado e ser um instrumento que contribuirá com a prevenção de sua saúde.

\section{Conclusão}

O estudo tratou do desenvolvimento de um aplicativo móvel denominado UP GLICEMIA para a monitorização dos níveis glicêmicos intermitentes por glicemia capilar e contínuos por sensor subcutâneo, de fácil manuseio, acessibilidade e compreensão para o indivíduo com diabetes tipo 1 e 2. Esta ação gerou um módulo de registro gráfico para a monitorização 
desses níveis, que se apresenta de forma individualizada e/ou simultânea. A construção do aplicativo UP GLICEMIA abre novos caminhos para o monitoramento do DM.

Espera-se que a utilização do UP GLICEMIA possa trazer um estímulo ao cuidado com a saúde do paciente com diabetes tipo 1 e tipo 2, por estimular a sua coparticipação no seu tratamento, possibilitando melhor entendimento do monitoramento da glicose, devido a favorecer o controle dos níveis glicêmicos bem como a prevenção de possíveis emergências, promovendo a melhoria da qualidade de vida. Por meio da utilização desse aplicativo, tanto o usuário quanto o profissional de saúde poderão ser beneficiados, possibilitando monitorizar o perfil glicêmico por completo.

Como trabalhos futuros, sugere-se a validação e avaliação do aplicativo, quanto a sua apresentação do conteúdo, funcionalidade e usabilidade com os especialistas e usuários bem como a comparação dos custos na utilização da tecnologia móvel no acompanhamento e forma tradicional de consultas.

\section{Referências}

Abbott. (2016). FreeStyler Libre. https://www.freestylelibre.com.br.

Alencar, I. G. M., Muniz, G. G., \& Medeiros, C. M. (2018). Monitorização glicêmica de adolescentes brasileiros com diabetes tipo 1. Revista de enfermagem UFPE. 12(7), 2012-2020.

Arrais, R. F., \& Crotti, P. L. R. (2015). Revisão: aplicativos para dispositivos móveis (“Apps”) na automonitorização em pacientes diabéticos. Journal of Health Informatics. 7(4), 127-133.

Arnhold, M., Quade, M., \& Kirch, W. (2014). Mobile applications for diabetics: a systematic review and expert-based usability evaluation considering the special requirements of diabetes patients age 50 years or older. J Med Internet Res. 16(4), e104.

Bellei, E. A., Biduski, D., Cechetti, N. P., \& De Marchi, A. C. B. (2018). Diabetes Mellitus in-Health Applications: A Systematic Review of Features and Fundamentals. Telemedicine and e-Health. 24(11):839-852. 10.1089/tmj.2017.0230.

Barra, D. C. C, Paim, S. M. S., Sasso, G. T. M. D., \& Colla, G. W. (2017). Métodos para desenvolvimento de aplicativos móveis em saúde: revisão integrativa de literatura. Texto Contexto Enfermagem, 26(4), 1-12.

Bessa, T., \& Arthaud, D. D. B. (2018). Metodologias ágeis para o desenvolvimento de softwares. Ciência e Sustentabilidade-CeS, 4(2), 173-213.

Boland, E. A., \& Tamborlane, V. W. (2000). Continuous glucose monitoring in youth with type 2 diabetes: overcoming barriers to successful treatment. Diabetes Technology Therapeutics. 2(1), 53-59.

Catalan, V. M., Denise, T. S., Agnes, L. N., Luísa, H. M. M., \& Gilberto, C. de M. B. (2011). Sistema NAS: nursingactivities score em tecnologia móvel. Revista da Escola de Enfermagem da USP. 45(6), 1419-1426.

Chiang, J. L., Kirkman, M. S., Laffel, L. M., \& Peters, A. L. (2014). Type 1 Diabetes Sourcebook Authors. Type 1 diabetes through the life span: a position statement of the American Diabetes Association. Diabetes Care, 37(7), 2034-2054.

Chomutare, T., Fernandez-Luque, L., Arsand, E., \& Hartvigsen, G. (2011). Features of mobile diabetes applications: review of the literature and analysis of current applications compared against evidence-based guidelines. Journal of Medical Internet Research, 13(3), 65-76.

Curioni, C. C., Brito, F. D. S. B., \& Boccolini, C. S. (2013). O uso de tecnologias de informação e comunicação na área da Nutrição. Jornal Brasileiro de TeleSSaúde. 2(3), 103-111.

Ferraz, D. P., Maia, F. F. R., \& Araújo, L. R. (2004). Glicemia capilar em ponta do dedo versus lóbulo de orelha: estudo comparativo dos valores resultantes e preferências dos pacientes. Arquivos Brasileiros de Endocrinologia \& Metabologia, 48, 389-393.

Ferreira, L. T., Saviolli, I. H., Valentini, V. E., \& Abreu, L. C. (2011). Diabetes melito: hiperglicemia crônica e suas complicações. Arquivo Brasileiro de Ciências e Saúde, 36(3), 182-188.

Geloneze, B., Lamounier, R. N., \& Coleho, O. R. (2006). Hiperglicemia pós-prandial: tratamento do seu potencial aterogênico. Arquivo brasileiro de cardiologia. 87(5), 660-670.

Goyotá, S. L. T., Chaves, E. de C. L. C., Andrade, M. B. T., Pereira, R. J. S., \& Brito, T. R. P. (2012). Ensino do processo de enfermagem a graduandos com apoio de tecnologias da informática. Acta paulista de enfermagem. São Paulo, 25(2), 243-248.

Guillen, S., Sanna, A., Ngo, J., Meneu, T., Hoyo, E., \& Demeester, M. (2009). New technologies for promoting a healthy diet and active living. Nutrition Reviews. 67(1), 100-107.

Hsu, W. C., Lau, K. H. K., Huang, R., Ghiloni, S., Le, H., Gilroy, S., Abrahamson, M., \& Moore, J. (2016). Utilization of a Cloud-Based Diabetes Management Program for Insulin Initiation and Titration Enables Collaborative Decision Making Between Healthcare Providers and Patients. Diabetes Technology Therapeutics, 18(2), 59-67. 
Insel, R. A., Dunne, J. L., Atkinson, M. A., Chiang, J. L., Dabelea, D., Gottlieb, P. A., Greenbaum, C. J., Herold, K. C., Krischer, J. P., Lernmark, A., Ratner, R E., Rewers, M. J., Schatz, D. A., Skyler, J. S., Sosenko, J. M., \& Ziegler, A. (2015). Staging presymptomatic type 1 diabetes: a scientific statement of JDRF, the Endocrine Society, and the American Diabetes Association. Diabetes Care, 38(10), 1964-1974.

Kaufman, F. R. (2000). Role of continuous glucose monitoring in pediatric patients. Diabetes Technology \& Therapeutics, 2(1), 49-52.

Leslier, R. D., Palmer, J., Schloot, N. C., \& Lernmark, A. (2016). Diabetes at the crossroads: relevance of disease classification to pathophysiology and treatment. Diabetologia, 59(1), 13-20.

Maia, M. M., Rodrigues, M. B., Morais, H. C. C., \& Oliveira, C. P. (2018). Informatização de diretriz para auxílio no diagnóstico por imagem de incidentalomas adrenais. Revista Expressão Católica Saúde, 3(1), 47-57.

Arnhold, M., Quade, M., \& Kirch. W. (2014). Mobile Applications for Diabetics: A Systematic Review and Expert-Based Usability Evaluation Considering the Special Requirements of Diabetes Patients Age 50 Years or Older. Journal of Medical Internet Research, 16(4), 104-122.

Martínez-Pérez, B., De La Torre-Díez, I., \& López-Coronado, M. (2013). Mobile health applications for the most prevalent conditions by the World Health Organization: review and analysis. Journal of Medical Internet Research, 15(6), e120.

Medivo. Ontrack Diabetes. (2015). https://play.google.com/store/apps/details?id=com.gexperts.ontrack\&hl=pt_BR

Nundy, S., Dick, J. J., \& Peek, M. E. (2013). Developing a behavioral model for mobile phone-based diabetes interventions. Patient Education and Counseling, 90(1), 125-132.

Oliveira, T. R., \& Costa, F. M. (2012). Desenvolvimento de aplicativo móvel de referência sobre vacinação no Brasil. Journal Health Information, 4(1), 23-27.

Pressmn, R. S. (2011). Engenharia de software: uma abordagem profissional. Bookman, (7a ed.), 87p.

Quasar. Telemedicina Gliconline. 2014.https://play.google.com/store/apps/details?id=br.com.quasar.gliconline\&hl=pt_BR

Queiroz, P.C., Aguia, D. C., Pinheiro, R. P., Moraes, C. C., Pimentel, I. R. S., Ferraz, C. L. H., \& Ferraz, T. M. B. L. (2011). Prevalência das complicações micro e macrovasculares e de seus fatores de risco em pacientes com diabetes mellitus e síndrome metabólica. Revista Brasileira de Clínica Médica, 9(4), 254258.

Queiroz, F. M., Aroldi, J. B. C., Oliveira, G. D. S., Peres, H. H. C., \& Vera Lúcia Conceição de Gouveia Santos, V. L. C. G. (2012). Úlcera venosa e terapia compressiva para enfermeiros: desenvolvimento de curso online*. Acta Paul Enferm, 25(3), 435-440.

Rossen, Varbanov. Diabetes $M$. 2015. https://play.google.com/store/apps/details?id=com.mydiabetes\&hl=pt_BR

SBD - Sociedade Brasileira de Diabetes. (2019). Posicionamento Oficial SBD no01/2019. https://www.diabetes.org.br/publico/images/pdf/sbd_dm2_ 2019_2.pdf

SBD - Sociedade Brasileira de Diabetes. (2019). Diretrizes da Sociedade Brasileira de Diabetes (2015-2016).

SBD - Sociedade Brasileira de Diabetes. (2017). Diretrizes da Sociedade Brasileira de Diabetes 2017-2018 / Organização José Egídio Paulo de Oliveira, Renan Magalhães Montenegro Junior, Sérgio Vencio. São Paulo: Editora Clannad, 383p.

SBD - Sociedade Brasileira de Diabetes. (2018). Diagnóstico e Tratamento. http://www.diabetes.org.br/ publico/diabetes/diagnostico-e-tratamento.

SBD - Sociedade Brasileira de Diabetes. (2020). Diretrizes da Sociedade Brasileira de Diabetes 2019-2020. Editora Clannad.

Squaremed Software Gmbh. (2015). Diabetes Plus. https://play.google.com/store/apps/details?id=com.squaremed.diabetesplus.typ1\&hl=pt_BR.

Tibes, C. M., Dias, J. D., \& Zem-Mascarenhas, S. H. (2014). Aplicativos móveis desenvolvidos para a área da saúde no Brasil: revisão integrativa da literatura . Revista Mineira de Enfermagem, 18(2), 479-486.

OMS - Organização Mundial da Saúde. (2016). Global reporton diabetes. https://apps.who.int/iris/bitstream/handle/10665/204871/9789241565257_eng.pd $\mathrm{f}$,jsessionid=4C745C0 0C4E7B93587C EB298 68FFE131? sequence $=1$.

Zimmet, P., Alberti, K. G., \& Shaw, J. (2001). Global and societal implicationsofthe diabetes epidemic. Nature, 414(6865), $782-787$. 